\title{
Hyperreal Delta Functions as a New General Tool for Modeling Systems with Infinitely High Densities
}

\author{
Marcoen J. T. F. Cabbolet
}

check for updates

Citation: Cabbolet, M.J.T.F.

Hyperreal Delta Functions as a New General Tool for Modeling Systems with Infinitely High Densities. Axioms 2021, 10, 244. https://doi.org/ 10.3390 /axioms 10040244

Academic Editors: Giovanni Calvaruso and Hans J. Haubold

Received: 20 June 2021

Accepted: 24 September 2021

Published: 29 September 2021

Publisher's Note: MDPI stays neutral with regard to jurisdictional claims in published maps and institutional affiliations.

Copyright: (C) 2021 by the author. Licensee MDPI, Basel, Switzerland. This article is an open access article distributed under the terms and conditions of the Creative Commons Attribution (CC BY) license (https:// creativecommons.org/licenses/by/ $4.0 /)$.
Center for Logic and Philosophy of Science, Free University of Brussels, Pleinlaan 2, 1050 Brussels, Belgium; Marcoen.Cabbolet@vub.be

\begin{abstract}
In general, the state of a system in which a physical quantity such as mass is distributed over space can be modeled by a function that represents the density distribution. The purpose of this paper is to introduce special functions that can be applied when in the system to be modeled, where the quantity is distributed over isolated points. For that matter, the expanded real numbers are introduced as an ordered subring of the hyperreal number field that does not contain any infinitesimals, and hyperreal delta functions are defined as special functions from the real numbers to the expanded real numbers satisfying the condition that (i) the support is a singleton, and (ii) the integral over the reals is a nonzero real number. These newly defined hyperreal delta functions, and tensor products thereof, then provide a general tool, applicable for the mathematical modeling of physical systems in which infinitely high densities occur.
\end{abstract}

Keywords: mathematical modeling; dirac delta; hyperreal numbers; systems theory

MSC: 33E20

\section{Introduction}

In physics, we are interested in modeling a state of a system in which a physical quantity is distributed over space. Let us limit the discussion to one-dimensional physical systems for the sake of simplicity, and suppose then that a real amount $\xi$ of a physical quantity (e.g., mass) is distributed over the space $\mathbb{R}$ : this state can then, in general, be modeled by a function $f: \mathbb{R} \rightarrow \mathbb{R}$, satisfying $\int_{-\infty}^{\infty} f(x) d x=\xi$, with the function value $f(x) \in \mathbb{R}$ at a point $x \in \mathbb{R}$ representing the numerical value of a density. However, troubles arise when the physical quantity is distributed over isolated points $x_{1}, x_{2}, \ldots, x_{n}$ in the space $\mathbb{R}$ : we have this situation, for example, when we consider the distribution of mass in a system made up of $n$ point particles. In such cases, infinitely high densities occur at those isolated points $x_{j} \in \mathbb{R}$ : no real function $f$ exists that can model such a state.

To model states of systems in which infinitely high densities occur, functions $f$ are required that can have an infinitely large value at isolated points in space, but such that $f$ can also be added to a (piecewise) smooth function $g$ representing a distribution of an amount $\chi$ of that physical quantity over a region of space-a sum $f+g$ then represents a distribution of an amount $\xi+\chi$ of that physical quantity over space. Analyzing, the problem is thus that functions $f$ are required that must satisfy the following two conditions:

$$
\begin{aligned}
& \text { (i) } f: \mathbb{R} \rightarrow R \\
& \text { (ii) }\left\{\begin{array}{l}
f(x)=0 \Leftrightarrow x \neq r \\
\int_{-\infty}^{+\infty} f(x) d x=\xi
\end{array}\right.
\end{aligned}
$$

where $r, \xi \in \mathbb{R}$ and $R$ is a number ring containing the reals: $R \supset \mathbb{R}$. 
The purpose of this paper is to solve this problem-note that it is defined in terms of a Riemann integral: the Lebesque integral is of no interest in this context. The new mathematical result in this paper is that we will introduce ordinary functions, on the real line, that satisfy both clauses of Equation (1) - the predicate ordinary here means that the functions in question are binary relations that associate each element of the real numbers $\mathbb{R}$ to exactly one element of the number ring $R$.

\section{Motivation for Introducing New Definitions}

Our attention is drawn to the Dirac delta. At its original introduction, it was not exactly defined, but rather heuristically characterized as an object-denoted by the symbol $\delta$-for which

$$
\left\{\begin{array}{l}
\delta(x)=0 \Leftrightarrow x \neq 0 \\
\int_{-\infty}^{+\infty} \delta(x) d x=1
\end{array}\right.
$$

where $x$ is a real variable [1]. This corresponds to clause (ii) of Equation (1) for $r=0, \xi=1$. There is no function $f: \mathbb{R} \rightarrow \mathbb{R}$ that has these properties. In fact, Von Neumann dismissed the idea of the Dirac delta as "fiction" [2].

Since then, however, various objects that capture the idea of the Dirac delta have been rigorously defined within the framework of standard analysis; for an overview, see, e.g., [3]. An example is the Dirac delta distribution, a linear functional $\delta: \mathcal{D}(\mathbb{R}) \rightarrow \mathbb{R}$ on the space of test functions on $\mathbb{R}$ defined by $\langle\delta, f\rangle:=f(0)$ for all $f \in \mathcal{D}(\mathbb{R})$ [4]. Its definition is often written as

$$
\langle\delta, f\rangle=\int_{-\infty}^{+\infty} f(x) \delta(x) d x=f(0)
$$

but the objection is then that this is an abuse of notation since the term ' $\delta(x)^{\prime}$ ' in the integrand does not refer to an existing object. To meet that objection, the term $\delta(x)$ can be viewed as a weak limit of a sequence of functions $g_{n}: \mathbb{R} \rightarrow \mathbb{R}$, e.g., the functions $g_{n}$ given, for positive integers $n$, by $g_{n}(x)=n$ for $x \in\left(-\frac{1}{2 n}, \frac{1}{2 n}\right)$ and $g_{n}(x)=0$ else; we then obtain

$$
\int_{-\infty}^{+\infty} f(x) \delta(x) d x:=\int_{-\infty}^{+\infty} \lim _{n \rightarrow \infty} f(x) g_{n}(x) d x
$$

Still, this weak limit $\delta$ does not exist in the space of all functions from $\mathbb{R}$ to $\mathbb{R}$.

Another standard object that formalizes the idea of the Dirac delta is the Dirac measure, a real function $\delta_{0}: \mathcal{B}(\mathbb{R}) \rightarrow \mathbb{R}$ on the $\sigma$-algebra of Borel sets of $\mathbb{R}$ defined, for $X \in \mathcal{B}(\mathbb{R})$, by $\delta_{0}(X)=1$ if $0 \in X$ and $\delta_{0}(X)=0$ else [5]. The Lebesgue integral of a (measurable) function $f: \mathbb{R} \rightarrow \mathbb{R}$ against the measure $\delta_{0}$ then gives

$$
\int_{-\infty}^{+\infty} f(x) \delta_{0}(d x)=f(0)
$$

So, the Dirac measure corresponds to a Dirac delta distribution as defined above in a natural way, but it has to be emphasized that the Dirac measure has no Radon derivativethat is, the Dirac measure $\delta_{0}$ does not correspond to an ordinary function $\delta$ on the reals such that we have $\int_{\mathbb{R}} f(x) \delta_{0}(d x)=\int_{\mathbb{R}} f(x) \delta(x) d x$.

Other objects equivalent to the above distribution and measure have been introduced, but the point is this: although they all capture the idea of the Dirac delta, none of them actually define a function satisfying Equation (35). That is, the objects that have been defined in the framework of standard analysis to formalize the idea of the Dirac delta are

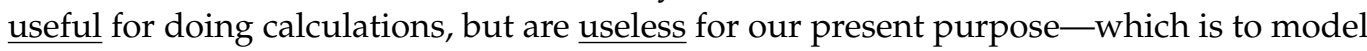
the state of a system in which a physical quantity is distributed over isolated points in space by a function on $\mathbb{R}^{n}$. 
Another development is that the real number field has been extended to the hyperreal number field $* \mathbb{R}[6]$. Todorov has shown that there exists a nonstandard (hyperreal) function ${ }^{*} \delta:{ }^{*} \mathbb{R} \rightarrow{ }^{*} \mathbb{R}$ such that

$$
\int_{* \mathbb{R}} f(x)^{*} \delta(x) d x=f(0)
$$

for any continuous function $f$ on $\mathbb{R}[7]$. An example is the function ${ }^{*} \delta:{ }^{*} \mathbb{R} \rightarrow{ }^{*} \mathbb{R}$ given by

$$
\left\{\begin{array}{l}
* \delta(x)=0 \Leftrightarrow x \notin\left[-\frac{1}{2 \omega}, \frac{1}{2 \omega}\right] \\
* \delta(x)=\omega \Leftrightarrow x \in\left[-\frac{1}{2 \omega}, \frac{1}{2 \omega}\right]
\end{array}\right.
$$

where $\omega$ is a hyperreal number-this and more examples can be found in [3]. So, this is an object defined in the framework of nonstandard analysis that captures the idea of the Dirac delta of Equation (35), but again this object is not useful for our present purposes: the point is that the function $* \delta$ violates condition (i) of Equation (1) because it is a function of a hyperreal variable, not of a real variable. This is objectionable, because in physics we want to model a spatial dimension with the real numbers, not with the hyperreal numbers.

Thus speaking, we cannot but conclude that the existing objects that formalize the idea of the Dirac delta are not suitable for our present purposes: we will, thus, have to develop a new object.

\section{Hyperreal Delta Functions of a Real Variable}

For our present purpose, we need hyperreal numbers, but we do not need all of the hyperreal number field. So, first of all, we apply Ockham's razor, and we define the ordered ring of the expanded reals as the part of the hyperreals that we need:

Definition 1. The ordered ring of expanded real numbers is the subring $\left({ }_{+}^{*} \mathbb{R},+, \cdot,>\right)$ of the hyperreal number field $\left({ }^{*} \mathbb{R},+, \cdot,>\right)$ given by

$$
{ }_{+}^{*} \mathbb{R}=\left\{\xi \in{ }^{*} \mathbb{R} \mid \xi=a_{1} \omega^{p_{1}}+\ldots+a_{n} \omega^{p_{n}}, n \in \mathbb{N}^{+}, p_{1}>\ldots>p_{n} \geq 0, a_{j} \in \mathbb{R}\right\}
$$

In words, the set ${ }_{+}^{*} \mathbb{R}$ contains, as indicated by the subscript ' + ' on the left, only those hyperreal numbers that can be written as a finite sum of a real number and products of a real number and a positive power of the infinitely large hyperreal number $\omega$ with $|\omega|=\infty$. If we define a real number as a set by identifying it with a Dedekind cut, then an expanded real number can be defined as a set by identifying it with the graph of a real polynomial:

$$
\sum_{j=1}^{n} a_{j} \omega^{p_{j}}:=\left\{(x, f(x)) \mid x \in \mathbb{R}, f(x)=\sum_{j=1}^{n} a_{j} x^{p_{j}}\right\}
$$

where $p_{1}>p_{2}>\ldots>p_{n} \geq 0$. As a corollary, the hyperreal number $\omega$ is then the set

$$
\omega:=\{(x, x) \mid x \in \mathbb{R}\}
$$

An inequality

$$
a_{1} \omega+a_{2} \neq a_{1} \omega
$$

is then an inequality of sets, that is, of graphs of polynomials. The ordering on the ring of expanded real numbers then coincides with the ordering on the ring $\mathbf{R}[t]$ of real polynomials described in [8].

Agreement 1. We will henceforth take the notation $\sum_{j=1}^{n} a_{j} \omega^{p_{j}}$ for an expanded real number $x \in{ }_{+}^{*} \mathbb{R}$ to imply that $p_{1}>p_{2}>\ldots>p_{n} \geq 0$ as in Definition 1 above.

The following two definitions are also useful. 
Definition 2. Let $x=\sum_{j=1}^{n} a_{j} \omega^{p_{j}}$ be an expanded real number. The real part of $x$ is then the number $\operatorname{Re}(x)$ given by

$$
\left\{\begin{array}{l}
p_{n}=0 \Rightarrow \operatorname{Re}(x)=a_{n} \\
p_{n}>0 \Rightarrow \operatorname{Re}(x)=0
\end{array}\right.
$$

Likewise, the hyperreal part of $x$ is then the number $H y(x)$ given by

$$
\left\{\begin{array}{l}
p_{n}=0 \Rightarrow H y(x)=x-a_{n} \\
p_{n}>0 \Rightarrow H y(x)=x
\end{array}\right.
$$

So, for any expanded real number $x$, we have $x=\operatorname{Re}(x)+H y(x)$.

Definition 3. Let $f: \mathbb{R} \rightarrow{ }_{+}^{*} \mathbb{R}$. Then, the real part of $f$ is the function $f_{R e}: \mathbb{R} \rightarrow{ }_{+}^{*} \mathbb{R}$ given by

$$
f_{R e}: x \mapsto \operatorname{Re}(f(x))
$$

Likewise, the hyperreal part of $f$ is the function $f_{H y}: \mathbb{R} \rightarrow{ }_{+}^{*} \mathbb{R}$ given by

$$
f_{H y}: x \mapsto H y(f(x))
$$

The space of all functions $f: \mathbb{R} \rightarrow{ }_{+}^{*} \mathbb{R}$ then forms a vector algebra over $\mathbb{R}$, when function addition, scalar multiplication, and function multiplication are defined naturally:

$$
\left\{\begin{array}{l}
(f+g)(x)=f(x)+g(x) \\
(\alpha \cdot f)(x)=\alpha f(x) \\
(f \cdot g)(x)=f(x) g(x)
\end{array}\right.
$$

In particular, for any $f: \mathbb{R} \rightarrow{ }_{+}^{*} \mathbb{R}$, we have $f=f_{R e}+f_{H y}$.

In this function space ${ }_{+}^{*} \mathbb{R}^{\mathbb{R}}$, we now define hyperreal delta functions $\alpha \delta_{\beta}$ as follows:

Definition 4. For any $\alpha, \beta \in \mathbb{R}$, the hyperreal delta function $\alpha \delta_{\beta}: \mathbb{R} \rightarrow{ }_{+}^{*} \mathbb{R}$ is a function given by

1. $x \neq \beta \Rightarrow \alpha \delta_{\beta}(x)=0$;

2. $x=\beta \Rightarrow \alpha \delta_{\beta}(x)=\alpha \omega$.

Note that for $f=\alpha \delta_{\beta}$, we have $f=f_{H y}$.

Our next step is that we define the hyperreal delta functions $\alpha \delta_{\beta}: \mathbb{R} \rightarrow{ }_{+}^{*} \mathbb{R}$ to have a real-valued integral over $\mathbb{R}$.

Definition 5. Let $\mathcal{R}^{1}(\mathbb{R})$ be the set of Riemann integrable functions on $\mathbb{R}$, and let the set of all integrable expanded real functions on $\mathbb{R}$ be denoted by ${ }_{+}^{*} \mathcal{R}^{1}(\mathbb{R})$. Let $f \in{ }_{+}^{*} \mathbb{R}^{\mathbb{R}}$; then $f \in{ }_{+}^{*} \mathcal{R}^{1}(\mathbb{R})$ if and only if

$$
\begin{gathered}
f_{\text {Re }} \in \mathcal{R}^{1}(\mathbb{R}) \\
f_{H y}=\sum_{n=1}^{\infty} \alpha_{n} \delta_{\beta_{n}}
\end{gathered}
$$

for some convergent series $\sum_{n=1}^{\infty} \alpha_{n}=s \in \mathbb{R}$; if $\int_{-\infty}^{+\infty} f_{R e}(x) d x=h$, then

$$
\int_{-\infty}^{+\infty} f(x) d x=\int_{-\infty}^{+\infty} f_{R e}(x) d x+\int_{-\infty}^{+\infty} f_{H y}(x) d x:=h+s
$$

Remark 1. Definition 5 has, thus, to be taken as an axiom of expanded real integral calculus: this does not "follow" from ordinary real integral calculus, nor can it be derived from the axioms of the hyperreal number field. 
Definition 5 thus says that an expanded real function $f$ on $\mathbb{R}$ is integrable if, and only if, the real part of $f$ is Riemann integrable and the hyperreal part of $f$ is a countable sum of hyperreal delta functions with the coefficients forming a convergent series.

Corollary 1. Writing $\delta_{0}$ for $1 \delta_{0}$, the function $\delta_{0}: \mathbb{R} \rightarrow{ }_{+}^{*} \mathbb{R}$ has all four essential characteristic properties of the Dirac delta identified by Lützen in [9], to wit:

1. $\delta_{0}(x)=\frac{d}{d x} H(x)$ with $H(x)$ the Heavyside function;

2. $\delta_{0}(x)=\lim _{n \rightarrow \infty} g_{n}(x)$ for suitable functions $g_{n}$;

3. $\delta_{0}(x)=0$ if $x \neq 0$ and $\int_{-\infty}^{\infty} \delta_{0}(x) d x=1$, cf. Equation (35);

4. $\quad \int_{-\infty}^{\infty} f(x) \delta_{0}(x) d x=f(0)$.

\section{Proof.}

1. For any closed interval $[a, b] \subset \mathbb{R}$, we have $\int_{a}^{b} \delta_{0}(x) d x=H(b)-H(a)$;

2. Consider the functions $g_{n}$ of Equation (4); then $\int_{-\infty}^{\infty} \delta_{0}(x) f(x) d x=\int_{-\infty}^{\infty} \lim _{n \rightarrow \infty} g_{n}(x) f(x) d x$ for any $f \in C^{\infty}(\mathbb{R})$;

3. This follows immediately from Definitions 4 and 5 ;

4. Writing $h(x)=f(x) \delta_{0}(x)$, note that $f(x) \delta_{0}(x)=h_{R e}(x)+h_{H y}(x)=0+f(0) \delta_{0}(x)$. Consequently, $\int_{-\infty}^{\infty} h(x) d x=\int_{-\infty}^{\infty} f(0) \delta_{0}(x) d x=f(0)$.

Theorem 1. Let $\mathbb{R}$ be the real numbers; then there is a set $S$ and an ordinary function $f: \mathbb{R} \rightarrow S$ such that $f$ has all properties of the Dirac delta.

Proof. This is true for $S={ }_{+}^{*} \mathbb{R}$ and $f=\delta_{0}$, cf. Corollary 1 .

As a set, any hyperreal delta function $\alpha \delta_{\beta}$ can thus simply be identified with its graph:

$$
\alpha \delta_{\beta}:=\left\{\langle x, \xi\rangle \in \mathbb{R} \times{ }_{+}^{*} \mathbb{R} \mid x \neq \beta \Rightarrow \xi=0 \wedge x=\beta \Rightarrow \xi=\alpha \omega\right\}
$$

The function $\delta_{0}$ in particular is thus an ordinary function on the reals with all the properties of the Dirac delta - the dismissal of the Dirac delta by Von Neumann was thus premature!

\section{Generalization}

Definition 5 can be generalized to integrable expanded real functions on $\mathbb{R}^{n}$. For that matter, we need the following definition:

Definition 6. Let $S_{n}$ be the group of permutations on $n$ letters. Let $\sigma \in S_{n}$. Then, a permutation of variables $\pi_{\sigma}:{ }_{+}^{*} \mathbb{R}^{\mathbb{R}^{n}} \rightarrow{ }_{+}^{*} \mathbb{R}^{\mathbb{R}^{n}}$ is a function on the space of all functions from $\mathbb{R}^{n}$ to ${ }_{+}^{*} \mathbb{R}$, such that the image $\pi_{\sigma}(f)$ of an arbitrary function $f: \mathbb{R}^{n} \rightarrow{ }_{+}^{*} \mathbb{R}$ under $\pi_{\sigma}$ is given by

$$
\pi_{\sigma}(f):\left(x^{1}, x^{2}, \ldots, x^{n}\right) \mapsto f\left(x^{\sigma(1)}, x^{\sigma(2)}, \ldots, x^{\sigma(n)}\right)
$$

Example 1. Let $\sigma \in S_{3}$ be the permutation $\left(\begin{array}{lll}1 & 2 & 3 \\ 2 & 3 & 1\end{array}\right)$, and let the function $f: \mathbb{R}^{3} \rightarrow{ }_{+}^{*} \mathbb{R}$ be given by $f:\left(x^{1}, x^{2}, x^{3}\right) \mapsto \sin \left(x^{1} x^{2}\right) \delta_{0}\left(x^{3}\right)$. Then, $\pi_{\sigma}$ is a permutation of variables such that

$$
\pi_{\sigma}(f):\left(x^{1}, x^{2}, x^{3}\right) \mapsto \sin \left(x^{2} x^{3}\right) \delta_{0}\left(x^{1}\right)
$$


Furthermore, we extend Definition 3 to expanded real functions on $\mathbb{R}^{n}$. That is, for any function $f: \mathbb{R}^{n} \rightarrow{ }_{+}^{*} \mathbb{R}$, the real and hyperreal parts of $f$ are the functions $f_{R e}$ and $f_{H y}$ given by

$$
\begin{aligned}
& f_{R e}:\left(x^{1}, \ldots, x^{n}\right) \mapsto \operatorname{Re}\left(f\left(x^{1}, \ldots, x^{n}\right)\right) \\
& f_{H y}:\left(x^{1}, \ldots, x^{n}\right) \mapsto H y\left(f\left(x^{1}, \ldots, x^{n}\right)\right)
\end{aligned}
$$

(Definition 3 can, of course, be extended to expanded real functions on any non-empty set $X$.)

Definition 7. Let $\mathcal{R}^{1}\left(\mathbb{R}^{n}\right)$ be the set of Riemann integrable functions on $\mathbb{R}^{n}$, and let the set of all integrable expanded real functions on $\mathbb{R}^{n}$ be denoted by ${ }_{+}^{*} \mathcal{R}^{1}\left(\mathbb{R}^{n}\right)$. Let, for positive integers $n, a$ symbol $\alpha \delta_{\left(\beta_{1}, \ldots, \beta_{n}\right)}^{n}$ denote a function $\alpha \delta_{\left(\beta_{1}, \ldots, \beta_{n}\right)}^{n}: \mathbb{R}^{n} \rightarrow{ }_{+}^{*} \mathbb{R}$ for which

$$
\alpha \delta_{\left(\beta_{1}, \ldots, \beta_{n}\right)}^{n}:\left(x^{1}, \ldots, x^{n}\right) \mapsto \alpha \delta_{\beta_{1}}\left(x^{1}\right) \delta_{\beta_{2}}\left(x^{2}\right) \cdots \delta_{\beta_{n}}\left(x^{n}\right)
$$

Let $f: \mathbb{R}^{n} \rightarrow{ }_{+}^{*} \mathbb{R}$; then $f \in{ }_{+}^{*} \mathcal{R}^{1}\left(\mathbb{R}^{n}\right)$ if and only if

$$
\begin{gathered}
f_{R e} \in \mathcal{R}^{1}\left(\mathbb{R}^{n}\right) \\
f_{H y}=\sum_{j=1}^{\infty} \pi_{\sigma_{j}} g_{j}
\end{gathered}
$$

where each $\pi_{\sigma_{j}}$ is a permutation of variables and each function $g_{j}$ satisfies

$$
g_{j}:\left(x^{1}, \ldots, x^{n}\right) \mapsto f_{n-k}\left(x^{1}, \ldots, x^{n-k}\right) \delta_{\left(\beta_{1}\left(x^{1}, \ldots, x^{n-k}\right), \ldots, \beta_{k}\left(x^{1}, \ldots, x^{n-k}\right)\right)}^{k}\left(x^{n-k+1}, \ldots, x^{n}\right)
$$

for some function $f_{n-k} \in \mathcal{R}^{1}\left(\mathbb{R}^{n-k}\right)$ —which for $k=n$ is a constant $f_{0} \in \mathbb{R}$-and the sum of the integrals of the $g_{j}$ 's is finite:

$$
\sum_{j=1}^{\infty} \int_{-\infty}^{\infty} \cdots \int_{-\infty}^{\infty} g_{j} d x^{1} \cdots d x^{n}=\sum_{j=1}^{\infty} s_{j} \in \mathbb{R}
$$

Example 2. Let $n=2$. Let the function $\alpha \delta_{\left(\beta_{1}, \beta_{2}\right)}^{2}: \mathbb{R}^{2} \rightarrow{ }_{+}^{*} \mathbb{R}$ be given by

$$
\alpha \delta_{\left(\beta_{1}, \beta_{2}\right)}^{2}:\left(x^{1}, x^{2}\right) \mapsto \alpha \delta_{\beta_{1}}\left(x^{1}\right) \delta_{\beta_{2}}\left(x^{2}\right)
$$

Then $\alpha \delta_{\left(\beta_{1}, \beta_{2}\right)}^{2}$ can be written as a tensor product:

$$
\alpha \delta_{\left(\beta_{1}, \beta_{2}\right)}^{2}=\alpha \delta_{\beta_{1}} \otimes 1 \delta_{\beta_{2}}
$$

For the integral of $\alpha \delta_{\left(\beta_{1}, \beta_{2}\right)}^{2}$ over $\mathbb{R}^{2}$, we then have

$$
\int_{-\infty}^{\infty} \int_{-\infty}^{\infty} \alpha \delta_{\left(\beta_{1}, \beta_{2}\right)}^{2}\left(x^{1}, x^{2}\right) d x^{1} d x^{2}=\int_{-\infty}^{\infty} \alpha \delta_{\beta_{1}}^{2}\left(x^{1}\right) d x^{1} \int_{-\infty}^{\infty} 1 \delta_{\beta_{2}}\left(x^{2}\right) d x^{2}=\alpha
$$

\section{Applications to Physics}

The hyperreal delta functions will be applied in the specification of the models of the Elementary Process Theory (EPT), a scheme of generalized process-physical principles governing the universe at Planck scale [10-12] —here, by a 'model', we mean a model in the sense of formal logic, see, e.g., $[13,14]$. With the newly defined hyperreal delta functions, constants of the EPT designating excited states and ground states of supersmall systems can be modeled by functions on the space-time manifold. A discrete state transition by 
which a supersmall system falls back from an excited state to the next ground state at Planck scale in $4 \mathrm{D}$ space-time can then be modeled by an expression

$$
E_{1} \delta_{\left(t_{1}, x_{1}, y_{1}, z_{1}\right)} \rightarrow E_{2} \delta_{\left(t_{2}, x_{2}, y_{2}, z_{2}\right)}
$$

for appropriate energies $E_{j}$ and spatiotemporal positions $\left(t_{j}, x_{j} \cdot y_{j}, z_{j}\right)$. The hyperreal delta functions in Expression (32) represent point-particle states, so such an expression-to be formalized as an $\in$-relation-then represents a discrete transition of the state of a Planckscale system made up of a single point particle.

However, the newly defined hyperreal delta functions can be applied in general to model physical systems in which a quantity is distributed over isolated points in space. For this mathematical modeling of physical systems, the newly introduced functions are advantageous compared to the existing objects that capture the idea of the Dirac delta: unlike these existing objects, the new functions enable one to model the state of a physical system in which infinitely high densities occur-such as the mass density in a system made up of point particles-by representing the distribution of the density over space with a function on $\mathbb{R}^{n}$.

Furthermore, writing $\delta_{\beta}^{n}(x)=\left(\delta_{\beta}(x)\right)^{n}$, we could initiate further developments based on Definition 5 by defining

$$
\int_{-\infty}^{\infty} \alpha \delta_{\beta}^{n}(x) d x=\alpha \omega^{n-1}
$$

so that

$$
\int_{-\infty}^{\infty} \delta_{0}^{2}(x) d x=\omega=\delta_{0}(0)
$$

We can apply this to non-relativistic quantum mechanics, where delta functions are routinely used as eigenstates of the position operator $\hat{x}$. In Dirac notation, a delta function $\delta(x-\alpha)$ with $\alpha \in \mathbb{R}$ is denoted $|\alpha\rangle$, and the inner product of two eigenfunctions of position $|\alpha\rangle$ and $|\beta\rangle$ is then

$$
\langle\alpha \mid \beta\rangle=\int_{-\infty}^{\infty} \delta(x-\alpha) \delta(x-\beta) d x=\delta(\alpha-\beta)
$$

See, e.g., [15]. This equation is on shaky foundations since the right-hand side does not have a well-defined numerical value if $\alpha=\beta$. However, if we apply our newly defined hyperreal delta functions, we obtain

$$
\begin{gathered}
|\alpha\rangle=\delta_{\alpha}(x) \\
\langle\alpha \mid \beta\rangle=\int_{-\infty}^{\infty} \delta_{\alpha}(x) \delta_{\beta}(x) d x=\delta_{0}(\alpha-\beta)
\end{gathered}
$$

Ergo, by applying the present definition of the Dirac delta as well as Equation (33), the inner product of two eigenvectors of the position as in Equation (35) takes a well-defined value in the expanded real numbers. This puts the use of the Dirac delta as a function on $\mathbb{R}$ in non-relativistic quantum mechanics on a rigorous mathematical footing while preserving its intended interpretation as a density distribution.

\section{Conclusions}

In conclusion, this paper has introduced hyperreal delta functions $\alpha \delta_{\beta}$ (with $\alpha, \beta \in \mathbb{R}$ ) as ordinary functions on the real line, such that, in particular, the hyperreal delta function $\delta_{0}$ (short for $1 \delta_{0}$ ) has all the properties of the Dirac delta. This is a new mathematical result, since existing objects that capture the idea of the Dirac delta are either generalized functions 
or functions on the hyperreal line. If we interpret the function value as a density, we can speak of infinitely high densities in those points where the value is nonzero, since $|\alpha \cdot \omega|=\infty$ for any $\alpha \in \mathbb{R}$. Consequently, expanded real functions $\alpha \delta_{\left(\beta_{1}, \ldots, \beta_{n}\right)}^{n}: \mathbb{R}^{n} \rightarrow{ }_{+}^{*} \mathbb{R}$, which can be written as a tensor product of $n$ hyperreal delta functions similar to the function $\alpha \delta_{\left(\beta_{1}, \beta_{2}\right)}$ in Equation (30), can henceforth all be called hyperreal delta functions-the special case $\delta_{0}$ can be called the Dirac delta function, as opposed to the Dirac delta distribution, defined according to Equation (3).

One might object that these new functions do not enable one to do previously impossible calculations or to prove new theorems in pure mathematics. However, that is not true. First of all, Theorem 1 is a new theorem in pure mathematics. Secondly, it has been shown that the present result can be applied to calculate the inner product of two eigenstates of the position operator in non-relativistic quantum mechanics: that is a calculation that, strictly speaking, was previously impossible. A true limitation of the present study is, however, that the set of integrable expanded-real functions on $\mathbb{R}$ is defined to be the set of expanded-real functions on $\mathbb{R}$ with a real-valued integral. An obvious generalization is to consider the set of expanded-real functions on $\mathbb{R}$ with a hyperreal-valued integral. A step in that direction has been suggested in Section 5 , but we leave the development of such a generalized definition of the set of integrable expanded-real functions on $\mathbb{R}$ as a topic for further research.

Funding: This research received no external funding.

Institutional Review Board Statement: Not applicable.

Informed Consent Statement: Not applicable.

Data Availability Statement: Not applicable.

Acknowledgments: This research has been facilitated by the Foundation Liberalitas.

Conflicts of Interest: The author declares no conflict of interest.

\section{References}

1. Dirac, P.A.M. The physical interpretation of the quantum dynamics. Proc. Roy. Soc. A 1927, 113, 621-641.

2. Von Neumann, J. Mathematische Grundlagen der Quantenmechanik; Springer: Berlin, Germany, 1932; p. 14.

3. Hoskins, R.P. Delta Functions: Introduction to Generalised Functions, 3rd ed.; Woodhead Publishing Limited: Cambridge, UK, 2009.

4. Schwartz, L. Theorie des Distributions. Tom I; Hermann: Berlin, Germany, 1950.

5. Rudin, W. Real and Complex Analysis; McGraw-Hill: New York, NY, USA, 1966.

6. Robinson, A. Non-Standard Analysis; Princeton University Press: Princeton, NJ, USA, 1966.

7. Todorov, T. A nonstandard delta function. Proc. Am. Math. Soc. 1990, 110, 1143-1144. [CrossRef]

8. Lang, S. Algebra, 3rd ed.; Springer: New York, NY, USA, 2002; p. 450.

9. Lützen, R. The Prehistory of the Theory of Distributions; Springer: New York, NY, USA, 1982; p. 130.

10. Cabbolet, M.J.T.F. Elementary Process Theory: a formal axiomatic system with a potential application as a foundational framework for physics supporting gravitational repulsion of matter and antimatter. Ann. Phys. 2010, 522, 699-738. [CrossRef]

11. Cabbolet, M.J.T.F. Addendum to the Elementary Process Theory. Ann. Phys. 2011, 523, 990-994. [CrossRef]

12. Cabbolet, M.J.T.F. Corrigendum to the Elementary Process Theory. Ann. Phys. 2016, 528, 626-627. [CrossRef]

13. Shoenfield, J.R. Mathematical Logic; AK Peters Ltd: Natick, MA, USA, 2001.

14. De Swart, H.C.M. Philosophical and Mathematical Logic; Springer: Berlin, Germany, 2018.

15. Griffith, D.J. Introduction to Quantum Mechanics; Prentice Hall: Englewood Cliffs, NJ, USA, 1994; p. 102. 